\title{
Prevalencia de desnutrición en niños y adolescentes en instituciones hospitalarias de América Latina: una revisión
}

\section{Prevalence of malnutrition in children and adolescents in hospitals in Latin America: a review}

\author{
Omar Danilo Ortiz-Beltrán ${ }^{1}$ (D) , Olga Lucia Pinzón-Espitia² (iD), Laura Brigitte Aya-Ramos³
}

1. Universidad Nacional de Colombia. Bogotá, Colombia. Correo: odortizb@unal.edu.co - https://orcid.org/0000-0003-2601-0410

2. Universidad Nacional de Colombia. Universidad del Rosario. Bogotá, Colombia. Correo: olpinzone@unal.edu.co - https://orcid.org/0000-0002-9827-

2244

3. Instituto Roosevelt. Bogotá, Colombia. Correo: Ibayar@unal.edu.co - https://orcid.org/0000-0002-8955-6798

Tipología: Artículo de revisión

Para citar este artículo: Ortiz-Beltrán OD, Pinzón-Espitia OL, Aya-Ramos LB. Prevalencia de desnutrición en niños y adolescentes en instituciones hospitalarias de América Latina: una revisión. Duazary. 2020 julio - septiembre; 17(3): 70 - 85. Doi: http://dx.doi.org/10.21676/2389783X.3315

Recibido en agosto 01 de 2019

Aceptado en noviembre 18 de 2019

Publicado en línea en abril 18 de 2020

\section{RESUMEN}

Palabras clave: desnutrición; trastornos de la nutrición del niño; hospital; evaluación; prevalencia

Keywords: Malnutrition; Child Nutrition Disorders; Hospital; Assessment; Prevalence.
Esta revisión buscó identificar la prevalencia de desnutrición en menores de 18 años hospitalizados en instituciones de América Latina, los criterios empleados para su clasificación, así como, la estancia hospitalaria y riesgo nutricional. Para esto, se realizó una búsqueda bibliográfica sistemática siguiendo la metodología PRISMA de artículos sobre prevalencia de desnutrición en niños y adolescentes relacionada con enfermedades en países latinoamericanos, publicados entre 1995 y enero del 2019. Fueron elegibles para su inclusión los estudios que informaron datos sobre la prevalencia de desnutrición en pacientes hospitalizados < de 18 años con un tamaño de muestra mayor a 50 sujetos. La revisión se llevó a cabo por tres revisores independientes que evaluaron la calidad metodológica. Como principal resultado se identifica que la prevalencia informada de desnutrición en pacientes pediátricos hospitalizados varía considerablemente. Esta cifra osciló entre el 3,3 y el $67 \%$. La diferencia se debe principalmente a la diversidad de las poblaciones evaluadas y a los métodos utilizados para detectar y evaluar el estado nutricional. Se tiene como principal conclusión que la elevada variabilidad reportada en cuanto a evaluación del estado nutricional plantea la necesidad de la unificación de estándares de clasificación que permitan favorecer la toma de decisiones a nivel hospitalario.

\section{ABSTRACT}

This review aims to describe the prevalence of child malnutrition in different hospitals in Latin America and to identify some of the indicators associated with the deterioration of nutritional status. To achieve this, a systematic bibliographic research was made following the PRISMA methodology, for articles on the prevalence of child and adolescents malnutrition related to diseases in Latin American countries published between 1995 and January 2019. Studies reporting data on prevalence in malnourished hospitalized patients $<18$ years with a sample size greater than 50 subjects were eligible for inclusion. The review was conducted by three independent reviewers who assessed methodological quality. As the main result, it was identified that the reported prevalence of malnutrition in hospitalized pediatric patients varies considerably. This figure ranged from 3,3 to $67 \%$. The difference is mainly due to the diversity of populations assessed and the methods used to detect and assess nutritional status. As a main conclusion, the high variability reported in terms of evaluation of nutritional status raises the need for the unification of classification standards to favor decision-making at the hospital level. 


\section{INTRODUCCIÓN}

El adecuado estado nutricional en la población infantil se relaciona con un buen estado de salud y se asocia con un óptimo potencial de crecimiento y desarrollo, contrario a esto, la desnutrición infantil se reconoce como uno de los factores responsables de un tercio de las muertes en menores de cinco años reportadas en la literatura, además de representar un problema de salud pública que demanda acciones de protección terapéuticas específicas $^{1-3}$.

Sumado a lo anterior, un estado de desnutrición en esta etapa tendrá un impacto negativo sobre el adecuado funcionamiento y desarrollo corporal, se puede asociar con disminución de la respuesta inmune del organismo, aumento ante la vulnerabilidad de enfermedades; además de representar un factor de riesgo para el desarrollo del cerebro y por ende de habilidades cognitivas y comportamentales en un futuro ${ }^{4-6}$.

A nivel hospitalario, Caldwell et $a l^{7}$ definen la desnutrición como un estado de deficiencia relativa o absoluta de uno o varios nutrientes y que se manifiesta clínicamente o es detectada por medio de exámenes bioquímicos o medidas antropométricas; constituyéndose desde hace décadas, un problema serio al interior de las instituciones encargadas de brindar cuidados médicos y asistenciales a largo plazo ${ }^{8}$.

Teniendo en cuenta lo anterior es posible diferir la desnutrición asociada a diferentes condiciones, una de tipo crónico donde existe carencia de los nutrientes necesarios durante un tiempo prolongado, caracterizada principalmente por un retraso en talla y una de tipo agudo determinada por un peso muy bajo para la talla, asociada en algunos casos con la pérdida severa de peso en poco tiempo y por ende la principal a nivel hospitalario y objeto de estudio de la presente revisión ${ }^{4,9}$.

Complementariamente, la Organización Mundial de la Salud hace referencia a las diversas causas que aumentan la probabilidad durante un proceso de hospitalización que conllevan a un deterioro nutricional en los niños, de igual manera se reconoce que la desnutrición durante la hospitalización puede tener importantes consecuencias poco favorables que por lo general pueden prolongar la estancia hospitalaria ${ }^{2}$. Pese a esto, en algunos países en vía de desarrollo, gran parte de los niños desnutridos pierden la oportunidad de ser diagnosticados en entornos clínicos, debido a la falta de mediciones de rutina de los índices antropométricos, particularmente la altura $^{10}$.

Sumado a esto, en ocasiones se omite la evaluación nutricional en algunos hospitales y por ende no se realiza de forma adecuada la terapia nutricional requerida, además de que la información sobre el estado nutricional en niños hospitalizados es limitada y existen menos estudios que reportan la desnutrición hospitalaria en población menor de 18 que en adultos ${ }^{10-12}$.

Por consiguiente, el realizar de forma oportuna y adecuada el diagnostico nutricional es de suma importancia en la atención clínica debido a que orienta la toma de decisiones; esto depende en gran medida de los criterios a utilizar, así como los referentes de crecimiento definidos por organizaciones internacionales ${ }^{13}$.

En Latinoamérica según la Organización Panamericana de la Salud (OPS) ${ }^{14}$ más de 2,5 millones de niños en América Latina y el Caribe con edades entre 0-36 meses presentan graves deficiencias de estatura y peso; esto sumado a la falta de identificación oportuna al interior de las instituciones hospitalarias puede incrementar las cifras de desnutrición y problemas vinculadas a esta.

Por consiguiente, la presente revisión tuvo por objetivo identificar la prevalencia de desnutrición en menores de 18 años hospitalizados en instituciones de América Latina, los criterios empleados para su clasificación, así como, la estancia hospitalaria y riesgo nutricional.

\section{MATERIALES Y MÉTODOS}

\section{Protocolo}

Se realizó una revisión sistemática de la literatura incluyendo análisis. Para garantizar la validez 
interna y externa, como la magnitud de los resultados, el protocolo de investigación cumplió con los aspectos conceptuales y metodológicos de la Declaración PRISMA. La población objetivo fueron niños y adolescentes menores de 18 años aleatoriamente seleccionados de la población general. No hubo intervención ni comparador de interés.

\section{Búsqueda}

La búsqueda bibliográfica se llevó a cabo en las siguientes bases de datos biomédicas: Biblioteca Virtual en salud (BVS), Cochrane Library, Medline (Medical Literature Analysis and Retrieval System Online), OVID Nursing y Web of Science, para artículos sobre prevalencia de desnutrición infantil reportada en instituciones hospitalarias de América Latina, publicados en inglés, español o portugués entre enero de 1995 y enero del 2019.

Los términos de búsqueda abarcaron cuatro categorías, enfermedad, región geográfica, resultados y población. Se incluyeron los artículos originales cuyo contenido permitiera la extracción de datos sobre prevalencia, resultados clínicos y asociación entre la condición de enfermedad y la desnutrición en población menor de 18 años, con un tamaño de muestra mayor de 50 sujetos. Se excluyeron revisiones narrativas, resúmenes sin artículo de texto completo, así como artículos sin información suficiente acerca de la población de estudio o los métodos analíticos.

Las palabras claves definieron la verificación en los descriptores de las ciencias de la salud MeSH y DECS: Malnutrition, Undernutrition, Malnourishment, Nutritional Deficiency, Hospital malnutrition, Disease related malnutrition, prevalence, length, stay, infections, comorbidity, mortality, weight loss, incidence, Latin America, South America, Central America Caribbean, child, children, pediatric hospital.

Como resultado de la conducción de la presente revisión se identificaron 1669 documentos, de los cuales 24 se consideraron potencialmente elegibles (Figura 1), complementariamente por búsqueda en literatura gris se identificaron 5 estudios adicionales.
Para la literatura gris, se hizo la búsqueda en el repositorio Institucional de la Universidad Nacional de Colombia y el buscador Google Escolar a partir de la ecuación de búsqueda: (Undernutrition OR Malnourishment OR Nutritional Deficiency) AND (Latin America OR South America OR Central America OR Caribbean) realizando la revisión en las primeras 10 páginas y haciendo una primera lectura del abstract para determinar su posible elegibilidad.

Todo lo anterior, permitió la obtención de 29 artículos, los cuales fueron analizados por los tres investigadores para definir su elegibilidad, encontrando que 20 artículos cumplieron con los criterios de calidad metodológica e inclusión definidos; población menor de 18 años, estudios con datos sobre prevalencia, riesgo de desnutrición o desnutrición relacionada a enfermedad en una población de estudio mayor a 50 sujetos en pacientes hospitalizados de países latinoamericanos publicados en inglés, español o portugués siendo estos metanálisis, revisiones sistemáticas, ensayos clínicos aleatorizados, estudios observacionales, estudios comparativos o series de casos.

La búsqueda en todas las bases de datos finalizó el $1 / 03 / 2019$, todos los resultados se consignaron en una matriz donde se registraba la fecha de búsqueda, la ecuación definida, los limitadores aplicados, el número de resultados obtenidos y las referencias seleccionadas, como ejemplo de esto la búsqueda en Medline se inicia el 2/2/2019 y finaliza el 24/2/19, se aplicó la ecuación de búsqueda definida con las cuatro categorías mencionadas, obteniendo un total de 539 resultados filtrados y revisados por "abstract", para un total de 18 potencialmente elegibles, sometidos posteriormente a lectura completa para evaluación de calidad metodológica y cumplimiento de todos los criterios de inclusión.

La extracción de la información, una vez seleccionados los estudios para la revisión, se realizó a través de una ficha de extracción desarrollada por consenso de los autores, la cual incluyó los ítems para responder al objetivo de la revisión sistemática. Para la evaluación de la calidad metodológica se hace uso del Instrumento de evaluación crítica para estudios que informan 
datos de prevalencia, el cual consta de 9 ítems que fueron sometidos a valoración por revisores independientes ${ }^{15}$.

Para la selección de artículos que establecieran la prevalencia de desnutrición se incluyeron aquellos que tuvieran en cuenta alguno los indicadores de crecimiento: Peso para la talla $(\mathrm{P} / \mathrm{T})$, talla para la edad $(T / E)$, peso para la edad $(P / E)$ e índice de masa corporal para la edad (IMC/E); comparados con estándares de tipo internacional dentro de las que están los definidos por la Organización mundial de la salud (OMS), El National Center for Health Statistics (NCHS) de los Estados Unidos de Norteamérica y las desarrolladas por Centers for Disease Control and Prevention (CDC $)^{16}$.

Adicionalmente, se incluyeron artículos que definieran la desnutrición con base al Índice de Waterlow modificado, el cual a partir de cifras actuales de peso, talla, edad y valores esperados según la media poblacional, permite la obtención de un porcentaje que posibilita definir y clasificar desnutrición ${ }^{17}$. Así mismo se incluyeron principalmente artículos de acceso libre y algunos de acceso restringido a los cuales se tuvo acceso por medio de los recursos bibliográficos de la Universidad Nacional de Colombia.

Los resultados del estudio para todas las publicaciones que cumplieron con los criterios de inclusión se resumen de manera descriptiva según la población de pacientes y los tipos de medición utilizados. Los datos de prevalencia basados en la clasificación antropométrica establecida por la OMS se informan como la proporción combinada de pacientes con desnutrición moderada y severa ${ }^{18}$.

Para lo anterior es necesario tener en cuenta que cada país adopta los parámetros definidos de forma internacional para clasificar la desnutrición, principalmente los establecidos por la OMS en el año 2006, los cuales se realizaron teniendo en cuenta una gran población de seis países, donde se determinó principalmente que existe un peso bajo para la talla, peso bajo para la edad y baja talla por debajo de-2 desviaciones estándar (DE) de la media poblacional. Como ejemplo de esto en Colombia se define desnutrición aguda por debajo de -2 DE y severa por debajo de $-3 \mathrm{DE}$ para el indicador $\mathrm{P} / \mathrm{T}^{19}$.

Para la prevalencia de riesgo con la herramienta STRONGKids, se establece el acumulado entre riesgo alto y medio, donde según la herramienta un puntaje de 0 se considera riesgo bajo, un puntaje de 1 a 3 riesgo medio y un puntaje de 4 o 5 riesgo alto $^{20}$.

\section{Riesgo de sesgo}

Los potenciales sesgos encontrados a realizar este trabajo en cuanto a la garantía de validez o veracidad, calidad metodológica y confiabilidad o reproducibilidad de resultados se encuentran enmarcados principalmente en diferencias en el tipo de estudio, rangos de edad variables y muy amplios en algunos casos, uso de indicadores antropométricos de diagnóstico diversos y métodos de clasificación de estado nutricional diferentes en algunos contextos. No obstante, dentro del proceso de selección se utilizaron estrategias para limitar estos sesgos y/o errores aleatorios mediante la búsqueda exhaustiva de los artículos más relevantes favoreciendo así la síntesis de los resultados obtenidos. Adicionalmente, los investigadores cuentan con la formación necesaria para una adecuada interpretación y análisis de los datos encontrados.

\section{RESULTADOS}

\section{Estudios identificados}

Como resultado de la conducción de la presente revisión se identificaron 1669 documentos, de los cuales 29 se consideraron potencialmente elegibles, estos fueron sometidos a evaluación metodológica dando un total de 20 artículos seleccionados. A continuación, se evidencia la metodología establecida para la búsqueda bibliográfica. 


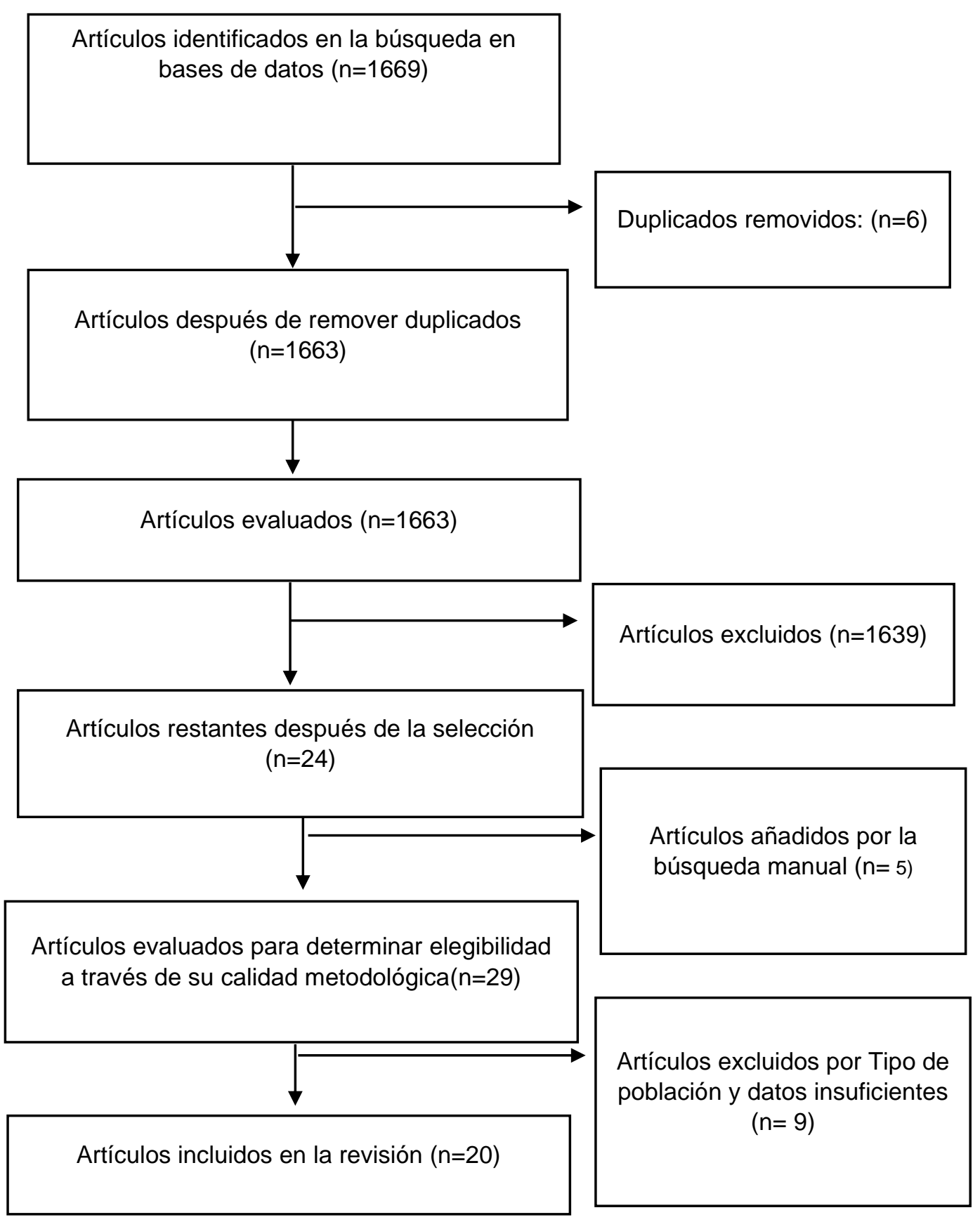

Figura 1. Diagrama de flujo de selección.

\section{Perfil de los estudios seleccionados}

Los estudios seleccionados en la presente revisión incluyeron pacientes de 10 países Latinoamericanos. Se evidenció que la mayoría de los estudios se realizaron en Brasil, representando el $34,3 \%$ de los pacientes incluidos en las diferentes poblaciones (Tabla 1). 
Tabla 1. Distribución de estudios y pacientes por país.

\begin{tabular}{|c|c|c|}
\hline País & Estudios & Pacientes (n) \\
\hline Argentina & 1 & 439 \\
\hline Brasil & 7 & 1899 \\
\hline Chile & 1 & 365 \\
\hline Colombia & 1 & 174 \\
\hline Cuba & 2 & 157 \\
\hline Haití & 1 & 278 \\
\hline México & 3 & 737 \\
\hline Paraguay & 2 & 748 \\
\hline El Salvador & 1 & 443 \\
\hline Nicaragua & 1 & 282 \\
\hline Total & 20 & 5522 \\
\hline
\end{tabular}

\section{Indicadores evaluación nutricional}

Seguido a esto, al realizar el análisis de los documentos se identificó que 15 estudios informaron datos de prevalencia de desnutrición en una población mixta o general, es decir que no se diferenció por el tipo de patología de base (Tabla 2) y en 5 estudios adicionales, la población objeto fueron pacientes con fibrosis quística y cáncer (Tabla 3).

Se encontró también que dentro de los parámetros definidos para evaluación nutricional se tuvieron en cuenta los diferentes indicadores asociados a los patrones de crecimiento infantil: Peso para la talla $(P / T)$, talla para la edad $(T / E)$, peso para la edad $(\mathrm{P} / \mathrm{E})$ e índice de masa corporal para la edad (IMC/E); estos fueron comparados con referentes de tipo internacional como las gráficas de referencia establecidas por la Organización Mundial de la Salud (OMS) las del National Center for Health Statistics (NCHS), CDC ${ }^{21}$.

\section{Riesgo Nutricional}

Adicionalmente, al realizar el análisis por riesgo nutricional, se encontró que tres estudios reportaron riesgo nutricional entre valores del $63,1 \%$ al $72 \%$ (Tabla 2), medido a través de la herramienta STRONGKids, la cual, a partir de 4 preguntas relacionadas con la enfermedad subyacente, evaluación clínica subjetiva, ingesta de alimentos y cambios de peso, establece el riesgo nutricional y la necesidad de intervención. A partir de una revisión sistemática se identifica a esta herramienta como la mejor para el tamizaje en pacientes pediátricos hospitalizados, dado que $63 \%$ de los estudios tenidos en cuenta en esta, refirieron que dicha herramienta es práctica, de fácil uso, altamente sensible pero no especifica y que permite detectar de forma fiable el riesgo de desnutrición ${ }^{22,23}$.

\section{Estancia hospitalaria}

Además, en relación con la estancia promedio de los pacientes objeto de estudio, se identificaron seis estudios que reportan cifras entre 4,5 y 9 días de hospitalización (Tabla 2). 
Tabla 2. Estudios que evalúan la prevalencia de desnutrición en pacientes menores de 18 años hospitalizados.

\begin{tabular}{|c|c|c|c|c|c|c|c|c|c|c|c|c|c|}
\hline Ref. & Año & País & $\begin{array}{c}\text { Grupo de } \\
\text { edad } \\
\text { (años) }\end{array}$ & Medidas & $N$ & $\begin{array}{l}\text { Método de } \\
\text { clasificación }\end{array}$ & $\begin{array}{l}\mathrm{T} / \mathrm{E} \\
\%\end{array}$ & $\begin{array}{l}\mathrm{P} / \mathrm{T} \\
\%\end{array}$ & $\begin{array}{l}\mathrm{IMC} / \mathrm{E} \\
\%\end{array}$ & $\begin{array}{l}\mathrm{P} / \mathrm{E} \\
\%\end{array}$ & $\%$ & $\begin{array}{l}\text { Promedi } \\
\text { o Días }\end{array}$ & $\begin{array}{l}\text { Tipo de } \\
\text { estudio }\end{array}$ \\
\hline \multirow{2}{*}{$\begin{array}{l}\text { Gomila et } \\
a^{24}\end{array}$} & \multirow[t]{2}{*}{2009} & \multirow[t]{2}{*}{ Argentina } & \multirow[t]{2}{*}{$0-6$} & $\begin{array}{c}\text { Peso, } \\
\text { Talla } \\
\text { PC }\end{array}$ & \multirow[t]{2}{*}{439} & $\begin{array}{l}\text { Waterlow } \\
\text { Modificado }\end{array}$ & 35 & 28 & - & 49,6 & \multirow[t]{2}{*}{-} & \multirow[t]{2}{*}{6} & \multirow{2}{*}{$\begin{array}{l}\text { Descriptivo, } \\
\text { analítico } \\
\text { prospectivo }\end{array}$} \\
\hline & & & & $\begin{array}{l}\mathrm{PCT} \\
\mathrm{CB} \\
\end{array}$ & & $<$ Percentil 3 & 19,7 & - & - & 25,1 & & & \\
\hline \multirow{2}{*}{$\begin{array}{l}\text { Vallandro } \\
\text { et a }{ }^{25}\end{array}$} & \multirow[t]{2}{*}{2018} & \multirow[t]{2}{*}{ Brasil } & \multirow[t]{2}{*}{$4-8,9$} & \multirow{2}{*}{$\begin{array}{l}\text { Peso } \\
\text { Talla }\end{array}$} & \multirow{2}{*}{447} & $\begin{array}{c}\text { OMS (z- } \\
\text { score) }<-2 \text { DE }\end{array}$ & 5,8 & - & 4 & - & - & - & \multirow{2}{*}{$\begin{array}{l}\text { Estudio } \\
\text { Transversal }\end{array}$} \\
\hline & & & & & & \multicolumn{5}{|c|}{$\begin{array}{c}\text { Riesgo nutricional } \\
\text { STRONGKids\% }\end{array}$} & 72,2 & - & \\
\hline $\begin{array}{l}\text { Moraes } \\
\text { et } a l^{26}\end{array}$ & 2008 & Brasil & $0-12$ & $\begin{array}{l}\text { Peso } \\
\text { Talla }\end{array}$ & 426 & $\begin{array}{c}\text { OMS (1995- } \\
2006)\end{array}$ & 21 & 10 & 14,7 & 18 & - & 2 & $\begin{array}{l}\text { Estudio de } \\
\text { cohorte }\end{array}$ \\
\hline $\begin{array}{l}\text { Gilberto } \\
\text { et } a 1^{27}\end{array}$ & 2000 & Brasil & $0-10$ & $\begin{array}{l}\text { Peso } \\
\text { Talla }\end{array}$ & 456 & OMS - 1995 & 17,1 & 10,7 & - & 20,4 & - & - & $\begin{array}{c}\text { Estudio } \\
\text { Prospectivo } \\
\end{array}$ \\
\hline \multirow{2}{*}{$\begin{array}{l}\text { Andrade } \\
\text { et } a^{28}\end{array}$} & \multirow{2}{*}{2016} & \multirow{2}{*}{ Brasil } & \multirow{2}{*}{$0-18$} & \multirow{2}{*}{$\begin{array}{l}\text { Peso } \\
\text { Talla }\end{array}$} & \multirow{2}{*}{122} & OMS 2006 & \multicolumn{4}{|c|}{$13,9 \%$} & \multirow{3}{*}{63,1} & \multirow[b]{2}{*}{8,4} & \multirow{2}{*}{$\begin{array}{l}\text { Estudio } \\
\text { transversal }\end{array}$} \\
\hline & & & & & & & $\begin{array}{l}\text { esgo n } \\
\text { STRON }\end{array}$ & $\begin{array}{l}\text { tricion } \\
\text { iKids\% }\end{array}$ & & & & & \\
\hline $\begin{array}{l}\text { Pileggi et } \\
a^{29}\end{array}$ & 2015 & Brasil & $0-18$ & $\begin{array}{l}\text { Peso } \\
\text { Talla }\end{array}$ & 292 & OMS & - & - & 16,1 & - & & - & $\begin{array}{l}\text { Estudio } \\
\text { transversal }\end{array}$ \\
\hline \multirow{2}{*}{$\begin{array}{l}\text { Oliviera } \\
\text { et } a^{30}\end{array}$} & \multirow{2}{*}{2017} & \multirow{2}{*}{ Brasil } & \multirow{2}{*}{$0-18$} & \multirow{2}{*}{$\begin{array}{c}\text { Peso } \\
\text { talla } \\
\text { PB }\end{array}$} & \multirow{2}{*}{71} & OMS & 12 & 9,7 & 7 & 9,6 & 60 & & Estudio \\
\hline & & & & & & & $\begin{array}{l}\text { esgo n } \\
\text { STRON }\end{array}$ & $\begin{array}{l}\text { tricion } \\
\text { iKids\% }\end{array}$ & & & 69 & - & \\
\hline $\begin{array}{l}\text { Velandia } \\
\text { et } a^{131} \\
\end{array}$ & 2016 & Chile & $0-17$ & $\begin{array}{l}\text { Peso } \\
\text { Talla } \\
\end{array}$ & 365 & OMS - 2006 & 12 & & 3 & & - & - & $\begin{array}{c}\text { Estudio } \\
\text { transversal }\end{array}$ \\
\hline $\begin{array}{l}\text { Sánchez } \\
\text { et } a^{32}\end{array}$ & 2012 & Colombia & $0-18$ & $\begin{array}{c}\text { Peso } \\
\text { Talla } \\
\text { PB }\end{array}$ & 174 & OMS - 2006 & 22,4 & 16,6 & - & 27 & - & 4,5 & $\begin{array}{l}\text { Estudio } \\
\text { transversal }\end{array}$ \\
\hline $\begin{array}{l}\text { Doirsanit } \\
\text { et } a^{\beta 3}\end{array}$ & 2018 & Haití & $0-2$ & $\begin{array}{l}\text { Peso } \\
\text { Talla }\end{array}$ & 278 & OMS & 13,3 & 16,7 & - & 18,4 & - & - & $\begin{array}{l}\text { Estudio } \\
\text { prospectivo }\end{array}$ \\
\hline $\begin{array}{l}\text { Rosales } \\
\text { et } a l^{\beta 4}\end{array}$ & 2009 & México & $0-15$ & $\begin{array}{l}\text { Peso } \\
\text { Talla }\end{array}$ & 626 & OMS & 17 & 8 & & & - & - & $\begin{array}{l}\text { Estudio } \\
\text { transversal }\end{array}$ \\
\hline $\begin{array}{l}\text { Valero et } \\
a^{\beta 5}\end{array}$ & 2011 & Cuba & $0-18$ & $\begin{array}{l}\text { Peso } \\
\text { Talla }\end{array}$ & 157 & $\begin{array}{c}\text { OMS } \\
(z-S C O R E)<-1\end{array}$ & 7,6 & & 19,7 & 10,2 & - & - & $\begin{array}{c}\text { Estudio } \\
\text { descriptivo } \\
\text { prospectivo }\end{array}$ \\
\hline $\begin{array}{l}\text { Maciques } \\
\text { et a }{ }^{36}\end{array}$ & 2014 & Cuba & $0-18$ & $\begin{array}{l}\text { Peso } \\
\text { Talla }\end{array}$ & $\begin{array}{c}195 \\
1\end{array}$ & OMS & & & 12,1 & & - & - & $\begin{array}{c}\text { Estudio } \\
\text { descriptivo } \\
\text { transversal }\end{array}$ \\
\hline $\begin{array}{l}\text { Sanabria } \\
\text { et } a^{37}\end{array}$ & 2000 & Paraguay & $0-18$ & $\begin{array}{l}\text { Peso } \\
\text { Talla }\end{array}$ & 493 & OMS & & 13,3 & & 30,6 & - & - & $\begin{array}{c}\text { Estudio } \\
\text { descriptivo } \\
\text { transversal }\end{array}$ \\
\hline $\begin{array}{c}\text { Acevedo } \\
\text { et } a^{\beta 8}\end{array}$ & 2005 & Paraguay & $0-18$ & $\begin{array}{l}\text { Peso } \\
\text { Talla }\end{array}$ & 255 & $\begin{array}{c}\text { OMS } \\
\text { Alguno de } \\
(\mathrm{P} / \mathrm{T}, \mathrm{T} / \mathrm{E}, \\
\mathrm{IMC} / \mathrm{E})<-2 \\
\end{array}$ & & & 14 & & - & 9 & $\begin{array}{c}\text { Estudio } \\
\text { descriptivo } \\
\text { transversal }\end{array}$ \\
\hline
\end{tabular}

PB: perímetro de brazo, P/E: peso para la edad, P/T: peso para la talla, T/E: talla para la edad, IMC/E: índice de masa corporal para la edad, CMB: circunferencia muscular del brazo, DE: desviaciones estándar, OMS: Organización Mundial de la Salud. 
Tabla 3. Prevalencia de desnutrición relacionada con algunas patologías.

\begin{tabular}{|c|c|c|c|c|c|c|c|}
\hline \multicolumn{8}{|c|}{ Fibrosis quística } \\
\hline $\begin{array}{l}\text { Irving } \\
\text { et }\left.a\right|^{39}\end{array}$ & 2011 & Brasil & $6-18$ & $\begin{array}{l}\text { Peso } \\
\text { Talla } \\
\text { PB } \\
\text { PC } \\
\text { PCT } \\
\text { CB }\end{array}$ & 85 & $\begin{array}{l}\text { Gráficas } \\
\text { de CDC }\end{array}$ & $\begin{array}{l}\text { IMC/E: } \\
22,3 \%\end{array}$ \\
\hline \multicolumn{8}{|c|}{ Cáncer } \\
\hline $\begin{array}{l}\text { Pedrosa } \\
\text { et } a l^{40}\end{array}$ & 2000 & $\begin{array}{c}\text { El } \\
\text { salvador }\end{array}$ & $0-18$ & $\begin{array}{l}\text { Peso } \\
\text { talla }\end{array}$ & 443 & $\begin{array}{l}\text { OMS- } \\
1995\end{array}$ & $\begin{array}{l}\text { P/E:23,5\% } \\
\text { P/T:15,7\% } \\
\text { T/E:22,8\% }\end{array}$ \\
\hline $\begin{array}{l}\text { Trejo } \\
\text { et } a l^{41}\end{array}$ & 2017 & México & $0-17$ & $\begin{array}{l}\text { Peso } \\
\text { Talla }\end{array}$ & 782 & OMS & $\begin{array}{l}\text { P/E: } 6,1 \% \\
\text { P/T: 7,5\% } \\
\text { T/E: } 10,6 \%\end{array}$ \\
\hline $\begin{array}{c}\text { Pribnow } \\
\text { et } a l^{42}\end{array}$ & 2017 & Nicaragua & $0-18$ & $\begin{array}{l}\text { Peso } \\
\text { Talla } \\
\text { PCT } \\
\text { CB } \\
\end{array}$ & 282 & OMS & $\begin{array}{l}\mathrm{PCT}<\mathrm{P} 10 \mathrm{o} \\
\mathrm{CB}<\mathrm{P} 5 \circ \\
\mathrm{IMC} / \mathrm{E}<\mathrm{P3} \\
=67 \%\end{array}$ \\
\hline $\begin{array}{l}\text { Conner } \\
\text { et } a l^{43}\end{array}$ & 2016 & México & $0-18$ & $\begin{array}{l}\text { Peso } \\
\text { Talla }\end{array}$ & 111 & OMS 2006 & $\begin{array}{l}\text { IMC / E: } \\
14,0 \%\end{array}$ \\
\hline
\end{tabular}

\section{DISCUSIÓN}

El rango de prevalencia de desnutrición reportado por los diferentes artículos mostró una alta variabilidad, esto debido principalmente a grandes diferencias entre los grupos evaluados, además de las diferentes técnicas empleadas para la evaluación del estado nutricional. La totalidad de los estudios se realizaron en centros de atención terciaria, incluyendo un amplio rango de edad (1-18 años) y se utilizaron diferentes tipos de indicadores asociados al estado nutricional.

En primer lugar, el rango de prevalencia de bajo peso para la talla $(\mathrm{P} / \mathrm{T})$ se encontró entre 8 y $16,7 \%$. Este mismo indicador evaluado por el método de Waterlow modificado mostró una prevalencia de desnutrición de $28 \%$, siendo el valor más alto de todos los artículos identificados. Dicho método ha sido utilizado por algunos investigadores para clasificar el estado nutricional basado en el porcentaje del peso estándar para la talla y el porcentaje de talla para la edad; adicionalmente este método permite determinar la cronología y la intensidad de la desnutrición basado principalmente en las medidas antropométricas que debería tener cada individuo.

Teniendo en cuenta lo anterior, es evidente el grado de población general afectada dado que sólo 2 de los 12 estudios que evaluaron este indicador presentaron un valor por debajo del 10\%, presentando cifras cercanas a este.

Al comparar estas cifras con las identificadas por otros estudios realizados en países fuera de Latinoamérica, fue posible identificar cifras similares; una revisión que reunió datos de prevalencia de desnutrición de 15 países europeos y 2 estudios realizados en Canadá mostró para el indicador $\mathrm{P} / \mathrm{T}$, un rango entre 9 y 14,8\%, usando como parámetro el encontrarse por debajo de dos desviaciones estándar ${ }^{44}$.

Del mismo modo, algunos estudios analizados de forma individual evidenciaron cifras con prevalecía similares. Un estudio realizado en Francia con una población de 280 individuos entre las edades de 2 
meses a 16 años, mostro una prevalencia de $11 \%$ de población por debajo de -2DE para el indicador $\mathrm{P} / \mathrm{T}^{45}$. Así mismo otro estudio realizado en el reino unido con una población de 255 individuos entre los 3 meses y los 18 años, presentó una prevalencia de desnutrición de $14 \%{ }^{46}$, siendo evaluada con los mismos parámetros, finalmente otro estudio de Holanda con una población de 424 individuos entre 1 mes y 18 años arrojó una prevalencia de bajo peso para la talla de $11 \%{ }^{47}$.

Lo anterior, pone en claro que el bajo peso para la talla a nivel hospitalario es un problema multifactorial, que, si bien es preocupante en países de recursos bajos debido al acceso limitado de alimentos o atención médica, también puede desencadenarse por otras causas como enfermedades en países en transición ${ }^{44,48}$.

Por otra parte, para el indicador de $\mathrm{IMC} / \mathrm{E}$, se identificó una población por debajo de dos desviaciones estándar un porcentaje entre $4 \mathrm{y}$ $16,10 \%$ de la población. Otro de los estudios mostró un IMC bajo, a la población que se encontraba por debajo de -1 desviaciones estándar, arrojando una prevalencia de $19,7 \%$.

Los resultados anteriores evidencian un comportamiento similar al encontrado para $\mathrm{P} / \mathrm{T}$, el cual también puede asociarse con una pérdida reciente de peso o emaciación y nos da claros indicios del estado nutricional, siendo este el parámetro principal a tener en cuenta en niños mayores de cinco años ${ }^{49,50}$.

Por otro lado, el indicador P/E se evaluó en 9 artículos de las citas identificadas, definiendo una prevalencia de bajo peso para la edad entre 9,6 y $30,7 \%$, además de esto, un estudio, mostró un valor de $49,6 \%$ mediante el método de Waterlow modificado.

Al compararlo con los demás indicadores, este presenta las cifras más elevadas de bajo peso, siendo este evaluado en niños menores de dos años en los cuales es clave el buen estado nutricional para un adecuado crecimiento $y$ desarrollo neurológico ${ }^{51}$.

Sin embargo, es válido aclarar que este indicador puede llegar a tener una interpretación compleja dada la influencia que tiene la talla en el peso, por lo cual puede llegar a evidenciar resultados diferentes; por tal motivo se sugiere ser tenido en cuenta para evaluar de forma poblacional y no individual $^{52}$.

Así mismo, al evaluar el indicador de talla para la edad se identifica una prevalencia de retraso en talla entre 5,8 y $22,4 \%$. Si bien el indicador presenta un comportamiento similar a los ya mencionados, no está asociado a una desnutrición debida a un periodo reciente de hospitalización; en cambio revela niños con retardo en el crecimiento coligado a diversas causas de las cuales podemos identificar las de tipo económico, políticas, culturales y sociales; siendo la pobreza el principal determinante asociado a una alimentación insuficiente $\mathrm{e}^{53}$.

Para este indicador, Latinoamérica demuestra cifras de desnutrición crónica en menores de cinco años en $12,2 \%{ }^{54}$. Esto puede estar vinculado a la desigualdad de la concentración económica a nivel interno medida con los niveles de ingresos de los hogares además de la desigualdad entre los países que la conforman ${ }^{55}$.

Otros aspectos identificados fueron los estudios que analizaron la prevalencia de desnutrición asociada a enfermedades como fibrosis Quística, con una prevalencia de bajo peso de $22,3 \%$ según el indicador IMC/E. Además de esto, en cáncer se encontraron valores de bajo peso entre 7,5 y $15,7 \%$ según $P / T$. Si bien son enfermedades que condicionan a estancias hospitalarias, se ven más asociadas a una condición crónica que favorece un deterioro en el estado nutricional ${ }^{56}$.

En relación al promedio de días de estancia hospitalaria, los estudios identificados evidenciaron un promedio de 6,7 días con un rango entre 4,5 y 9 días. Cinco estudios de las citas identificadas concuerdan en que un deterioro en el estado nutricional previo al ingreso incrementa la estancia hospitalaria y favorece la recuperación tardía ${ }^{26,31,32}$.

Sumado a lo anterior, algunos autores relacionan el deterioro del estado nutricional con el aumento de la estancia hospitalaria, esto es asociado a las complicaciones relacionadas con la enfermedad o a condiciones propias del proceso de hospitalización, soportando la importancia de un diagnostico 
nutricional oportuno al interior de estas instituciones ${ }^{11,26,27,31}$.

Por otra parte, tres estudios de las citas identificadas reportaron la medición del riesgo nutricional mediante la herramienta STRONGKids, reportando cifras entre 63,1 y 72,2 ; esto evidencia que más de la mitad de la población evaluada en estos casos requerían de intervención nutricional oportuna ${ }^{25,25,30}$.

Igualmente, un estudio realizado bajo un contexto hospitalario identificó que el tamizaje nutricional permitió la identificación temprana en $49 \%$ de los pacientes desnutridos ${ }^{57}$, esto sumado a lo anterior, evidencia la importancia de incluir el tamizaje nutricional en las diferentes instituciones que prestan servicios asistenciales a nivel de América Latina donde las cifras de riesgo fueron elevadas.

Los valores de prevalencia de desnutrición identificados, dependiendo del tipo de indicador, pueden asociarse con las condiciones inherentes al proceso de hospitalización; en el cual tanto los procesos patológicos, como los medicamentos administrados repercuten de forma negativa en el estado nutricional; estos últimos alteran el metabolismo de nutrientes y condicionan a una menor ingesta de alimentos, esto contrasta con los altos requerimientos de calorías y nutrientes que no se cubren, provocando un balance negativo

Teniendo en cuenta lo anterior, además de las condiciones económicas ya mencionadas que repercuten de forma negativa el estado nutricional de los niños, se resalta la importancia de realizar una identificación oportuna del estado nutricional en instituciones hospitalarias de América Latina dada la importancia que tiene sobre el adecuado crecimiento y desarrollo infantil además de asociarse con una mejor oportunidad de mejora bajo un contexto hospitalario.

\section{CONCLUSIÓN}

La desnutrición hospitalaria en población pediatría es alta y varía entre países. Aunque en ocasiones es difícil de cuantificar debido a diversos estándares de medición, diferencias poblacionales, centros asistenciales con diferentes grados de especialización y patologías que se atienden, se entre lo consumido y lo demandado por el organismo ${ }^{58}$.

De forma más específica, es importante resaltar que algunos estudios demuestran que el peso bajo para la talla o desnutrición aguda en algunos casos ocurre de manera frecuente bajo circunstancias de hospitalización donde las condiciones asociadas afectan la pérdida de peso en periodos cortos de tiempo ${ }^{59}$.

Mcglone et $a l^{60}$ hicieron una relación de las posibles causas de la desnutrición hospitalaria dentro de las que se encontraban el tipo de enfermedad, que con frecuencia tiende a interferir con el apetito y la capacidad del paciente para hacer el manejo de las diferentes comidas. Además de eso se señala que la apariencia o palatabilidad de la comida conjunto con la rutina hospitalaria pueden ir en contra de los hábitos de alimentación del paciente.

Bajo este contexto son múltiples las causas que llevan a que un paciente que ingresa a un servicio de hospitalización se encuentre en un elevado riesgo de presentar desnutrición en un muy corto periodo de tiempo, ya sea por la condición de base, los síntomas asociados a esta, la alteración fisiológica del metabolismo o la interferencia de la hospitalización en los hábitos alimentarios del paciente ${ }^{61}$.

estima que esta es una problemática general que necesita intervención oportuna para garantizar una adecuada recuperación clínica de los pacientes. Su importancia radica en que el riesgo de morir es directamente proporcional a la gravedad de la desnutrición, y esto se asocia a su vez a mayor gravedad de la enfermedad.

De igual manera, es bien sabido que los pacientes desnutridos presentan más complicaciones durante su hospitalización como, por ejemplo: peor evolución clínica, necesidad de asistencia respiratoria más prolongada, peor cicatrización de heridas y mayor riesgo de infecciones.

De acuerdo con lo anterior, a nivel hospitalario las condiciones asociadas a la hospitalización favorecen e incrementan el riesgo de un deterioro a nivel nutricional, es por ello que el detectar el riesgo nutricional merece de principal atención por 
parte de las instituciones hospitalarias, debido al impacto que tiene en el tiempo de estancia hospitalaria y por ende los costos de los servicios de salud.

Así mismo, la presente revisión identifica las altas cifras asociadas a la prevalencia de desnutrición hospitalaria en niños y la necesidad de implementar programas que consideren la intervención nutricional como uno de los ejes principales en la intervención del paciente dada la importancia que tiene en el adecuado crecimiento y desarrollo de los niños.

\section{RECOMENDACIONES}

La elevada variabilidad reportada en cuanto a evaluación del estado nutricional plantea la necesidad de la unificación de estándares de clasificación que permitan llevar a cabo proyectos de investigación que permitan la comparación entre poblaciones, con el fin de favorecer la toma de decisiones a nivel hospitalario.

Así mismo se sugiere identificar la prevalencia de desnutrición en todos los hospitales con población menor de 18 años con el fin de establecer un panorama inicial de las condiciones nutricionales de los pacientes y establecer medidas que permitan controlar y mejorar dicha situación.

El incorporar herramientas de tamizaje nutricional en los protocolos de evaluación nutricional de pacientes pediátricos hospitalizados, resulta de gran importancia en países Latinoamericanos debido a condiciones de tipo socioeconómico que sumadas a los factores que favorecen el deterioro nutricional a nivel hospitalario aumentan la probabilidad de desnutrición de los pacientes pediátricos y por ende las consecuencias que ejerce sobre un adecuado crecimiento y desarrollo.

\section{DECLARACIÓN SOBRE CONFLICTOS DE INTERESES}

Los autores no presentan ningún conflicto de intereses relacionado con el tema de la presente investigación, todos los autores participaron en el diseño, análisis e interpretación de datos.

\section{CONTRIBUCIÓN DE LOS AUTORES}

Primer autor: Búsqueda bibliográfica, análisis de resultados y redacción.

Segundo autor: Concepción de la investigación, construcción de objetivos y metodología, revisión de artículos, análisis de resultados y revisora metodológica.

Tercer autor: Revisión de artículos, redacción, análisis de resultados y revisora metodológica.

\section{REFERENCIAS BILIOGRAFICAS}

1. Martínez C. Malnutrición infantil en el medio hospitalario. Jornadas Nacionales del Centenario de la Sociedad Argentina de Pediatría Gastroenterología, Hepatología y Nutrición Pediátricas. Mendoza; 2011. Disponible en https://www.sap.org.ar/docs/congresos/2011/ GHN/Resumenes/martinezcostamalnutricion.pd $f$

2. Organización Mundial de la Salud. Fomento de una alimentación adecuada del lactante y del niño pequeño [Internet]. Organización Mundial de la Salud. 2019; Disponible en: https://www.who.int/nutrition/topics/infantfee ding/es/

3. Enrique N, Ortiz A. Desnutrição E Bioética: Reflexões Sobre Um Problema De Saúde Pública Malnutrition and Bioethics: Reflections on a Public Health Problem. 2012;22:28-35. Disponible en: http://www.scielo.org.co/scielo.php?script=sci_ arttext\&pid=S165747022012000100003\&lng=en

4. Unicef. Causas, consecuencias y estrategias para su prevención y tratamiento. UOC Nutr [Internet]. 2011; 36. Disponible en: https://www.unicef.es/sites/unicef.es/files/com unicacion/Informe_La_desnutricion_infantil.pdf

5. Paredes-Arturo YV. Chronic malnutrition and cognitive performance. Univ y Salud. 2015; 17(1):47-56. Disponible en: http://www.scielo.org.co/scielo.php?script=sci_ arttext\&pid=S0124-

$71072015000100005 \& \operatorname{lng}=$ en. 
6. Organización Mundial de la Salud. Temas de salud Nutrición [Internet]. [Consultado 2019 June 11]. Disponible en: https://www.who.int/topics/nutrition/es/

7. Caldwell MD, Kennedy-Caldwell C. Normal nutritional requirements. Surg Clin North Am [Internet]. 1981; 61(3):489-507. Doi: https://doi.org/10.1016/s0039-6109(16)424321

8. Zúñiga AES, Torres GFH, Chávez YJ, Olguín GQ, Meza EM. Guía de práctica clínica. Desnutrición intrahospitalaria: Tamizaje, diagnóstico y tratamiento. Guía Práctica Clínica. 2013; Disponible en: http://www.imss.gob.mx/sites/all/statics/guiasc linicas/641GER.pdf

9. Fondo de las Naciones Unidas para la Infancia UNICEF. Lineamiento para el manejo integrado. Unicef; $2017 . \quad$ Disponible en: https://www.minsalud.gov.co/sites/rid/Lists/Bib liotecaDigital/RIDE/VS/PP/lineamientodesnutricion-aguda-minsalud-unicef-final.pdf

10.Antwi S. Malnutrition: Missed opportunities for diagnosis. Ghana Med J. 2011; 42(3). Disponible en:

https://www.ncbi.nlm.nih.gov/pmc/articles/PM C2643431/

11.JPM, Pennington $\mathrm{C}$. Incidence and recognition of malnutrition in hospital. Br Med J 1994;308:9458. $\mathrm{Br}$ Med J [Internet]. 1994;308:945-8. Disponible en: https://www.ncbi.nlm.nih.gov/pmc/articles/PM C2539799/pdf/bmj00435-0025.pdf

12.Castro-Amador A, Matamoros M. Valoración Nutricional Intrahospitalaria En Niños Del Hospital Materno Infantil E Instituto Hondureno De Seguridad Social REV MED POST UNAH SeptDic. 2000;5(3):211-7. Disponible en: http://cidbimena.desastres.hn/RMP/pdf/2000/ pdf/Vol5-3-2000-4.pdf

13.Moreno Villares JM, Varea Calderón V, Bousoño García C. Malnutrición en el niño ingresado en un hospital. Resultados de una encuesta nacional. An Pediatr. 2017; 86(5):270-6. Doi: https://doi.org/10.1016/j.anpedi.2015.12.013
14.Vio F. Hacia la erradicación de la desnutrición infantil en América Latina y el Caribe. Capítulo 1. La desnutrición infantil en Chile: políticas y programas que explican su erradicación. Conferencia Regional Ministerial Santiago de Chile, 2008 [Internet]. 2008. Disponible en: http://www.odaalc.org/documentos/1370991060.pdf

15.The Joanna Briggs Institute. Checklist for Systematic Reviews and Research Syntheses. Joanna Briggs Inst [Internet]. 2016;1-7. Disponible en: http://joannabriggs.org/research/criticalappraisal-tools.html

16.Gonzalez MA, Pino JL. Estudio comparativo de las curvas de crecimiento NCHS/OMS: Evaluación del estado nutricional e implicancias en un centro de salud familiar. Rev Chil Nutr. 2010;37(2):169-77. Disponible en: https://scielo.conicyt.cl/scielo.php?script=sci_ar ttext\&pid=S071775182010000200005\&lng=esht tp://dx.doi.org/10.4067/S071775182010000200005

17.Abril C. Valoración nutricional mediante curvas de crecimiento de la oms y las clasificaciones de gómez/waterlow. Estudio de prevalencia. Rev Fac Cienc Méd Univ Cuenca Diciembre [Internet]. 2015;33(3):65-74. Disponible en: http://dspace.ucuenca.edu.ec/jspui/bitstream/1 23456789/25147/1/Karla Chacón Abril\%2C José Segarra Ortega.pdf

18.Organización Mundial de la Salud. Interpretando los Indicadores de Crecimiento [sede Web]. Ginebra- Suiza: Organización Mundial de la Salud; 2008 [acceso 04 de junio de 2018]. [Internet]. 2009. Disponible en: http://www.who.int/childgrowth/training/c_int erpretando.pdf

19.De Onis M, Garza C, Onyango AW, Borghi E. Comparison of the WHO child growth standards and the CDC 2000 growth charts. Acta Paediatr. 2006;95(450):106.

Doi: https://doi.org/10.1093/jn/137.1.144

20.García-Rivera R, Montijo-Barrios E, CervantesBustamante R, Zárate-Mondragón F, CadenaLeón JF, Toro-Monjaraz EM, et al. Screening 
tools in hospitalized children Correspondencia. Acta Pediátrica México [Internet]. 2018;39(4):338-51 Disponible en: www.actapediatrica.org.mx

21.Álvarez Castaño LS, Estrada Restrepo A, Goez Rueda JD, Carreño Aguirre C. Análisis de datos antropométricos de la población menor de 18 años de Medellín usando los estándares de la Organización Mundial de la Salud y su adaptación para Colombia propuesta por el Ministerio de la Protección Social TT - Analysis of anthropometric. Perspect en Nutr Humana [Internet]. 2012;14(1):33-45. Disponible en: http://www.scielo.org.co/pdf/penh/v14n1/v14n 1a4.pdf

22.Patane J, Jereb S. Descripción de las herramientas de tamizaje nutricional en el paciente pediátrico. Diaeta [Internet]. 2016; Disponible en: http://www.scielo.org.ar/scielo.php?script=sci_ arttext\&pid=S1852-73372016000300005

23.Aponte Borda AD, Pinzón Espitia OL, Aguilera Otalvaro PA. Tamizaje nutricional en paciente pediátrico hospitalizado: revisión sistemática. Nutr Hosp. 2018;35(5):1221. Doi: https://doi.org/10.20960/nh.1658

24.Gomila AA, Elizabeth De Grandis DS, Graciela Visconti LB, Susana Montero DB, Marta Bertero LI, Graciela Marietti L, et al. Artículo original Estado nutricional en niños internados en Salas de Cuidados Mínimos. Hospital de Niños de la Santísima Trinidad. Córdoba. Arch Argent Pediatr. 2009; 107(1):37-42. Disponible en: https://pesquisa.bvsalud.org/portal/resource/pt /lil-515401

25.Vallandro JP, da Silveira Klein Campos L, Neumann LD, de Mello ED. Adductor muscle thickness of the thumb: A new and reliable parameter for nutritional assessment of pediatric inpatients. Clin Nutr [Internet]. 2018;(February):1-6. Doi: https://doi.org/10.1016/j.clnu.2018.02.010

26.De Moraes Silveira CR, De Mello ED, Antonacci Carvalho PR. Evolution of nutritional status of pediatric in patients of a tertiary care general hospital in Brazil. Nutr Hosp. 2008; 23(6):599606. https://dialnet.unirioja.es/servlet/articulo?codig $o=2757484$

27.Gilberto K, Patricia C, Silva D, Silveira R, Boas V, Pinhiero A. Lenght of stay is an associated with incidence of in hospital malnutrition in a group of low-income Brazilian Children. Salud Pública de Mexico; 2000.2 Doi: https://doi.org/10.1590/s003636342000000500006

28.Andrade M, Nery MZ, Oliveira A, Santos B. Nutritional risk and associated factors in hospitalized paediatric patients through the STRONGKids. Nutr Clin y Diet Hosp. 2016; 36(2):158-67.

Doi: https://doi.org/10.12873/362zildamoura

29.Pileggi VN, Monteiro JP, Margutti AVB, Camelo JS. Prevalence of child malnutrition at a university hospital using the World Health Organization criteria and bioelectrical impedance data. Brazilian J Med Biol Res. 2016; 49(3):1-8. Doi: https://doi.org/10.1590/1414$431 \times 20155012$

30.Oliveira, TCD, Albuquerque IZD, Stringhini MLF, Mortoza AS, Morais BAD. The nutritional status of hospitalized children and adolescents: a comparison between two nutritional assessment tools with anthropometric parameters. Revista paulista de pediatria: orgao oficial da Sociedade de Pediatria de Sao Paulo. 2017; 35(3): 273-280. Doi: http://dx.doi.org/10.1590/19840462/;2017;35;3;00006

31.Velandia S, Hodgson MI, Le Roy C. Evaluación nutricional en niños hospitalizados en un Servicio de Pediatría. Rev Chil Pediatr. 2016;87(5):359-65.

Doi: http://dx.doi.org/10.1016/j.rchipe.2016.05.001

32.Sánchez LRS, Rodríguez DAS, Galarza ALV. Nutritional status among hospitalized children with mixed diagnoses at a referral teaching hospital in Manizales, Colombia. 2012;1451-9. Doi: https://doi.org/10.3305/nh.2012.27.5.5875

33.Doirsaint R, Bustos E, Irarrázaval B, Barja S, Senethmm G, Uauy R, et al. Influence of Feeding Practices on Malnutrition in Haitian Infants and 
Young Children. Nutrients. 2018; 10(3):382. Doi: https://doi.org/10.3390/nu10030382

34.Macías-Rosales $R$, Vásquez-Garibay $E$, LarrosaHaro A, Rojo-Chávez M, Bernal-Virgen A, RomoRubio $H$. Secondary Malnutrition and Overweight in a Pediatric Referral Hospital: Associated Factors. Journal of Pediatric Gastroenterology and Nutrition. 2009; 48(2):226-232. Disponible en: http://ovidsp.ovid.com/ovidweb.cgi?T=JS\&PAGE $=$ reference $\& D=$ med $5 \& N E W S=N \& A N=19179886$ \%5Cnhttp://ovidsp.ovid.com/ovidweb.cgi?T=JS \&PAGE $=$ reference \&D $=$ emed $9 \& N E W S=N \& A N=20$ 09368008

35.Valero WR, Zirena I, Coronel M, Dueñas JR. Estado Nutricional de los niños atendidos en el hospital pediátrico "Juan Manuel Márquez", de la Habana Cuba. Acta Med Per. 2009; 26(3):1515. Disponible en: https://www.medigraphic.com/cgibin/new/resumen.cgi?IDARTICULO=49491

36. Maciques Rodríguez R, Alfonso Novo L, Jiménez García R, Senra Reyes LM, Rodríguez Hernández $E$, Cordero Herrera $M$, et al. Frecuencia de desnutrición pediátrica en hospitales de Cuba. Acta Pediatr Esp. 2014; 72(11):e384-8. Disponible en: http://www.actapediatrica.com/index.php/secci ones/nutricion-infantil/1054-frecuencia-dedesnutricion-pediatrica-en-hospitales-decuba\#.XcTOidVKhOw

37.Sanabria M, Dietz E, Achucarro C. Evaluación nutricional de niños hospitalizados en un servicio de pediatría de referencia. Pediatria [Internet]. 2000 [cited 3 March 2019];(27):16$23 . \quad$ Disponible en: https://www.revistaspp.org/index.php/pediatri a/article/view/373/343

38.Acevedo E, Sanabria M, Bellenzier A, Baruja D, Buongermini $A$, Meza $R$, et al. EVolución del estado nutricional de pacientes pediátricos hospitalizados. Pediatr. (Asunción) [Internet]. 2005 Dic [citado 2019 Jun 14]; 32(2): 12-18. Disponible en: http://scielo.iics.una.py/scielo.php?script=sci_ar ttext\&pid=S1683-98032005000200003\&lng=es
39.Irving SY, Daly B, Verger J, Typpo K V., Brown AM, Hanlon $A$, et al. The Association of Nutrition Status Expressed as Body Mass Index Z score With Outcomes in Children with Severe Sepsis: A Secondary Analysis from the Sepsis Prevalence, Outcomes, and Therapies (SPROUT) Study. Crit Care Med. 2018;46(11):e1029-39. Doi:

https://doi.org/10.1097/CCM.00000000000033 51

40.Pedrosa F, Bonilla M, Liu A, Smith K, Davis D, Ribeiro RC, et al. Effect of malnutrition at the time of diagnosis on the survival of children treated for cancer in El Salvador and Northern Brazil. Am J Pediatr Hematol Oncol. 2000; 22(6):502-5.

Doi: https://doi.org/10.1097/00043426-20001100000005

41.Martín-Trejo JA, Núñez-Enríquez JC, FajardoGutiérrez A, Medina-Sansón A, Flores-Lujano J, Jiménez-Hernández $E$, et al. Early mortality in children with acute lymphoblastic leukemia in a developing country: the role of malnutrition at diagnosis. A multicenter cohort MIGICCL study. Leuk Lymphoma. 2017; 58(4):898-908. Doi: https://doi.org/10.1080/10428194.2016.121990 4

42.Pribnow AK, Ortiz R, Báez LF, Mendieta L, LunaFineman $S$. Effects of malnutrition on treatmentrelated morbidity and survival of children with cancer in Nicaragua. Pediatr Blood Cancer. 2017; 64(11). Doi: https://doi.org/10.1002/pbc.26590

43.Conner JM, Aviles-Robles MJ, Asdahl PH, Zhang $\mathrm{FF}$, Ojha RP. Malnourishment and length of hospital stay among paediatric cancer patients with febrile neutropaenia: A developing country perspective. BMJ Support Palliat Care. 2016; 6(3):338-43.

Doi: https://doi.org/10.1136/bmjspcare-2015001020

44.McCarthy A, Delvin E, Marcil V, Belanger V, Marchand $V$, Boctor $D$, et al. Prevalence of Malnutrition in Pediatric Hospitals in Developed and In-Transition Countries: The Impact of Hospital Practices. Nutrients. 2019; 11(2):236. Doi: https://doi.org/10.3390/nu11020236 
45. Marteletti O, Caldari D, Guimber D, Mention K, Michaud L, Gottrand F. Dépistage de la malnutrition chez l'enfant hospitalisé: Influence de la structure d'accueil dans la prise en charge. Arch Pediatr. 2005; 12(8):1226-31. Doi: https://doi.org/10.1016/j.arcped.2005.02.020

46. Moy RJD, Smallman S, Booth IW. Malnutrition in a UK children's hospital. J Hum Nutr Diet. 1990; 3(2):93-100.

Doi: https://doi.org/10.1111/j.1365-

277X.1990.tb00056

47.Joosten KFM, Hulst JM. Prevalence of malnutrition in pediatric hospital patients. Curr Opin Pediatr. 2008; 20(5):590-6. Doi: https://doi.org/10.1097/MOP.0b013e32830c6e de

48.Kramer C, Allen S. Malnutrition in developing countries. Paediatr Child Heal (United Kingdom) [Internet]. 2015; 25(9):422-7. Doi: http://dx.doi.org/10.1016/j.paed.2015.04.002

49. Unicef. Evaluación del crecimiento de niños y niñas [Internet]. Material de apoyo para equipos de atención primaria de la salud. 2013. 11 p. Disponible en: http://files.unicef.org/argentina/spanish/Nutrici on_24julio.pdf?os+y+ni?as\#0

50.Abeyá E, Calvo E, Duran P, Longo E, Mazza C. Evaluación del estado nutricional de niñas, niño y embarazadas mediante antropometría [Internet]. Buenos Aires: Ministerio de Salud de la Nación, con el apoyo de la Organización Panamericana de la Salud (OPS/OMS); 2009 [cited 14 June 2019]. Disponible en: http://www.msal.gob.ar/images/stories/bes/gra ficos/0000000256cnt-a07-manual-evaluacionnutricional.pdf.

51.Quino AC, Barreto P. Desarrollo motor en niños con desnutrición en Tunja, Boyacá. Rev Fac Nac Salud Pública. 2015; 33(1):15-21. Disponible en: http://www.scielo.org.co/pdf/rfnsp/v33n1/v33n 1a03.pdf

52.Colombia Ministerio de Salud y Protección social. Colombia. Resolución Número 0002465 del 2016 por la cual se adoptan los indicadores antropométricos, patrones de referencia y puntos de corte para la clasificación antropométrica del estado nutricional de niñas, niños y adolescentes menores de 18 años de edad, adultos de 18 a 64 años de edad y gestantes adultas y se dictan otras disposiciones (junio 14 2016). Disponible en: http://www.icbf.gov.co/portal/page/portal/Port alICBF/bienestar/nutricion/pnsan/Resolucion 2465 de 2016.pdf

53.Angarita A, Prada GE, Mendoza GL, Hernández MN. Factores Socioeconómicos Asociados a Retraso En Talla En Preescolares Asistentes a Una Institución Educativa De Floridablanca, Colombia. Rev Chil Nutr [Internet]. 2016; 43(2):2-2. Disponible en: http://www.scielo.cl/scielo.php?script=sci_artte xt\&pid=S0717-

$75182016000200002 \& \operatorname{lng}=e n \& n r m=i s o \&$ tlng=e $\mathrm{n}$

54.Organización de las Naciones Unidas. Seguridad Alimentaria y Nutricional América Latina y el Caribe [Internet]. [Consultado 2019 Jun 1]. Disponible en: https://dds.cepal.org/san/estadisticas

55.Paraje G. Evolución de la desnutrición crónica infantil y su distribución socioeconómica en siete países de América Latina y el Caribe [Internet]. Serie políticas sociales. 2008. 66 p. Disponible en: http://repositorio.cepal.org/handle/11362/6145

56.García-Luna P, Parejo-Campos J, Pereira-Cunill J. Causas e impacto clínico de la desnutrición y caquexia en el paciente oncológico. Nutrición Hospitalaria [Internet]. 2006 [cited 26 August 2019];(21):10-16. Disponible en: http://scielo.isciii.es/scielo.php?script=sci_artte xt\&pid=S0212-16112006000600003\&Ing=es

57.Zanin R, Perdomo C, Palomar A. Utilidad de la evluación del estado nutricional en pacientes hospitalizados. Soc Iberomaericana Inf Cient [Internet]. 2011. Disponible en: https://www.siicsalud.com/dato/experto.php/1 23395\#esp

58.Waitzberg DL, Ravacci GR, Raslan M. Desnutrición hospitalaria. Nutr Hosp. 2011; 26(2):254-64. Disponible en: 
http://scielo.isciii.es/pdf/nh/v26n2/03_revision _01.pdf

59.Groleau V, Thibault M, Doyon M, Brochu EE, Roy CC, Babakissa C. Malnutrition in hospitalized children: Prevalence, impact, and management. Can J Diet Pract Res. 2014; 75(1):29-34. Doi: https://doi.org/10.3148/75.1.2014.29

60.Mcglone PJD. The feeding of patients in hospital : a review. Nutr Res Cent South Bank
Univ. $\quad 1994 ; 115(5): 282-8 ; \quad$ Doi: https://doi.org/10.1177/146642409511500504

61.Socarras $M$, Bolet $M$, Fernandez $T$, Morales $M$, Betanxourt D, Suarez R. Algunas causas que llevan a la desnutrición en los pacientes hospitalizados. Rev Cubana Med [Internet]. 2004; 43 (2-3). Disponible en: http://scielo.sld.cu/scielo.php?script=sci_arttext \&pid=S0034-75232004000200006 